Research Article

\title{
The Novel Regulatory Role of lncRNA-miRNA-mRNA Axis in Amyotrophic Lateral Sclerosis: An Integrated Bioinformatics Analysis
}

\author{
Dingsheng Liu, ${ }^{1}$ Xiaojia Zuo $\mathbb{D}^{1},{ }^{1}$ Peng Zhang, ${ }^{2}$ Rui Zhao, ${ }^{2}$ Donglin Lai, ${ }^{2}$ Kaijie Chen, ${ }^{2}$ \\ Yuru Han, ${ }^{2}$ Guoqing Wan, ${ }^{2}$ Yanjun Zheng $\mathbb{D}^{2}$, Changlian Lu $\mathbb{D}^{2}$, and Xuefeng Gu $\mathbb{D}^{2,3}$ \\ ${ }^{1}$ Department of Oncology and Hematology, Shanghai University of Medicine and Health Sciences Affiliated Zhoupu Hospital, \\ Shanghai, China \\ ${ }^{2}$ Shanghai Key Laboratory of Molecular Imaging, Shanghai University of Medicine and Health Sciences, Shanghai, China \\ ${ }^{3}$ Research Department, Shanghai University of Medicine and Health Sciences Affiliated Zhoupu Hospital, Shanghai, China
}

Correspondence should be addressed to Yanjun Zheng; zhengyj@sumhs.edu.cn, Changlian Lu; lvcl@sumhs.edu.cn, and Xuefeng Gu; guxf@sumhs.edu.cn

Received 8 January 2021; Revised 2 March 2021; Accepted 5 March 2021; Published 16 April 2021

Academic Editor: Lei Chen

Copyright (C) 2021 Dingsheng Liu et al. This is an open access article distributed under the Creative Commons Attribution License, which permits unrestricted use, distribution, and reproduction in any medium, provided the original work is properly cited.

\begin{abstract}
Amyotrophic lateral sclerosis (ALS) is an incurable neurodegenerative disease that primarily affects motor neurons, causing muscle atrophy, bulbar palsy, and pyramidal tract signs. However, the aetiology and pathogenesis of ALS have not been elucidated to date. In this study, a competitive endogenous RNA (ceRNA) network was constructed by analyzing the expression profiles of messenger RNAs (mRNAs) and long noncoding RNAs (lncRNAs) that were matched by 7 ALS samples and 4 control samples, and then a protein-protein interaction (PPI) network was constructed to identify the genes related to ALS. Gene Ontology (GO) was used to study the potential functions of differentially expressed mRNAs (DEmRNAs) in the ceRNA network. For the ALS and control groups, 247177 potential lncRNA-mRNA ceRNA relationship pairs were screened. Analysis of significant relationship pairs demonstrated that the PPI modules formed by the MALAT1-regulated SYNRG, ITSN2, PICALM, AP3B1, and AAK1 genes may play important roles in the pathogenesis of ALS, and these results may help to characterize the pathogenesis of ALS.
\end{abstract}

\section{Introduction}

Amyotrophic lateral sclerosis (ALS) is an incurable chronic neurological disease that can lead to the continuous death of upper and lower motor neurons, resulting in muscle atrophy and fatigue that affect the patient's limb movement until the death of the patient [1-4]. This disease is most likely to occur in middle-aged people, with an incidence rate of 1.52.7 per 100,000 people observed; in addition, most patients die within 5 years after the onset of the disease, which has a severe impact on the physical and mental health of patients $[1,5,6]$. Although the aetiology and pathogenesis of ALS have not been fully elucidated to date, they may be related to genetics, the immune/inflammatory response $[7,8]$, sphingolipid metabolism [9], oxidative stress [10], and glutamate excitotoxicity [5].
ALS includes two types: familial ALS and sporadic ALS. Familial ALS accounts for only 5\%-10\% of ALS cases, while sporadic ALS accounts for 90\%-95\% of cases [11]. Regardless of whether there is a family history, the disease pathogenesis is related to mutations in genes including SOD1 [7, 12], OPTN [6, 13], UBQLN2 [11], C9orf72 [8], SQSTM1 [2], SETX [2], GARP [9], PFN1 [14], and SPG7 [15] and genes that encode RNA-binding proteins [16], such as TARDBP, $h n R N P A 2 B 1, h n R N P A 1$, and FUS.

With the development of microarray and nextgeneration sequencing technologies, noncoding RNAs (ncRNAs) and other indirectly pathogenic genes have received extensive attention from researchers. Numerous studies [1, 17-23] have analysed long ncRNAs (lncRNAs), messenger RNAs (mRNAs), and microRNAs (miRNAs) in different specimens, such as serum and cerebrospinal fluid 
samples and muscle biopsies from ALS patients, and found that large numbers of miRNAs are differentially regulated. mRNAs, lncRNAs, and other RNA transcripts can act as endogenous miRNA sponges to inhibit miRNA functions. These interactions can be explained by the well-known competing endogenous RNA (ceRNA) hypothesis proposed by Salmena et al., which has been applied to many fields [24, 25]. Continued analysis of ceRNA networks may help to elucidate how different subtypes of ncRNAs interact.

In this study, we performed a comprehensive analysis of the mRNA and lncRNA expression profiles in ALS. Then, we constructed an ALS-specific ceRNA network using a large number of research objects from online databases. As far as we know, this study is the earliest to create an lncRNAmiRNA-mRNA ceRNA network in ALS. This study helps to characterize the molecular pathogenesis of ALS and thus provides promising clues for clinical treatment. Interestingly, the SYNRG, ITSN2, AAK1, PICALM, and AP3B1 modules in the protein-protein interaction (PPI) network, which are regulated by the lncRNA MALAT1, may play important roles in the pathogenesis of ALS.

\section{Materials and Methods}

2.1. Data Collection and Analysis. Data on miRNA-lncRNA regulatory relationships were downloaded from the experimental module of the lncBase database (http://carolina.imis.athenainnovation.gr/diana_tools/web/index.php? $r=\operatorname{lncbasev} 2 \% 2$ Findex), and miRNA-mRNA regulatory relationships were confirmed by experimental data downloaded from miRTarBase (http://mirtarbase.cuhk.edu.cn/php/index.php) to ensure the accuracy of miRNA target gene prediction. With regard to the potential ceRNA regulatory relationships between lncRNAs and mRNAs, if the number of common target miRNAs between lncRNAs and mRNAs was more than 3 and significance was indicated by a hypergeometric test, a potential ceRNA relationship between the lncRNAs and mRNAs was accepted. The equation for the hypergeometric test is shown in

$$
P=1-F(x \mid N, L, M)=1-\sum_{k=0}^{x-1} \frac{\left(\begin{array}{l}
L \\
k
\end{array}\right)\left(\begin{array}{c}
N-L \\
M-t
\end{array}\right)}{\left(\begin{array}{l}
N \\
M
\end{array}\right)}
$$

where $N$ represents the total number of miRNAs, $L$ represents the number of miRNAs targeting lncRNAs, $M$ represents the number of miRNAs targeting mRNAs, and $x$ represents the number of miRNAs targeting both $\operatorname{lncRNAs}$ and mRNAs. During screening, a $P<0.05$ was considered to indicate a potential ceRNA relationship between lncRNAs and mRNAs.

Sample RNA-seq data (GSE115259) were downloaded from the Gene Expression Omnibus (GEO, http://www .ncbi.nlm.nih.gov/geo/) database; the data were analysed by Illumina RNA-seq on peripheral blood mononuclear cells from 7 ALS patients (including sporadic and mutated ALS patients) and 4 controls. The lncRNAs in the extracted expression profile were considered to be the expressed
IncRNAs, and the expression profile was further filtered to move the mRNAs and lncRNAs expressed in more than half of the samples. Subsequent analysis was performed on the remaining lncRNAs and mRNAs. The correlation between lncRNAs and mRNAs was calculated by the R language cor.test function, and the correlation was calculated by the Spearman method. The lncRNA-mRNA regulatory relationships with $P<0.01$ and $R>0.5$ were screened.

2.2. Gene Ontology (GO) and Pathway Enrichment Analysis. The GO database is aimed at establishing a language vocabulary standard that strictly defines and comprehensively describes the gene and protein functions of any organization and can be dynamically updated with the continuous deepening of research. The GO system is an internationally standardized gene function classification system with three categories: molecular functions, cellular components, and biological processes.

In this study, the $\mathrm{R}$ language circlize package was used for visualization. The functions of network genes were analysed with Metascape.

2.3. PPI Network. All differentially expressed genes (DEGs) were imported into a search tool for identifying gene interactions, known as STRING 10.5 (https://string-db.org/) to construct the network, and Cytoscape software 3.6 (https://www .cytoscape.org) was employed for visualization. The colours of the edges in the network represent the types of proteinprotein relationships: light blue and purple show known interactions determined from the planning database and experiments, respectively; dark green/red/dark blue represents predicted interactions through gene neighbourhood/gene fusion/gene sharing, respectively; and light green/black/blue represents text mining/coexpression/protein homology.

2.4. Statistical Analysis. We used SPSS 11.0 (SPSS, Chicago, IL) to analyse the data sets from RNA-seq experiments. $P$ values $<0.05$ were considered to indicate significance.

\section{Results}

3.1. Construction of a ceRNA Network with ALS and Control Sample. We found that ALS samples and control samples were separated using $t$-Distributed Stochastic Neighbour Embedding $(t$-SNE) for dimensionality reduction visualization (Figure S1). Next, the target miRNAs of lncRNAs were obtained from the lncBase database, which consists of 100727 miRNA-lncRNA regulatory pairs composed of 1420 miRNAs and 8217 lncRNAs. Furthermore, the miRNA-mRNA target relationships were obtained from miRTarBase, which consists of 243613 pairs of miRNAmRNA target relationships consisting of 2585 miRNAs and 13618 mRNAs. Screening for a lncRNA-mRNA shared miRNA number greater than 3 and a significant result of the hypergeometric test $(P<0.05)$ left 247177 potential lncRNA-mRNA ceRNA relationship pairs (Table S1). The correlations between the lncRNA and mRNA expression in potential ceRNA pairs in ALS and the control samples were calculated, which revealed a significant lncRNA-mRNA ceRNA pair (Table S2), as shown in Figure 1. Figure 1(a) 


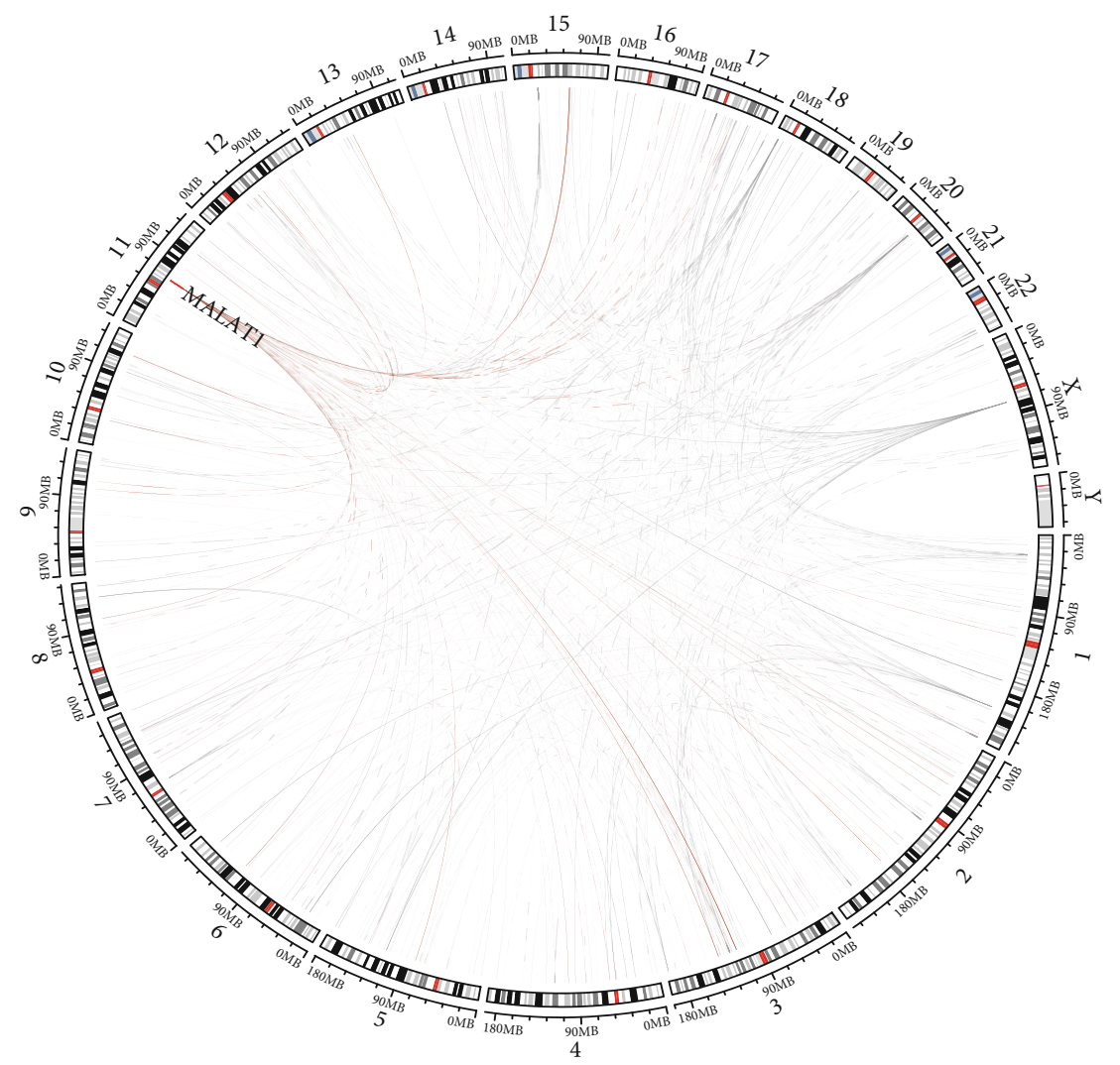

(a)

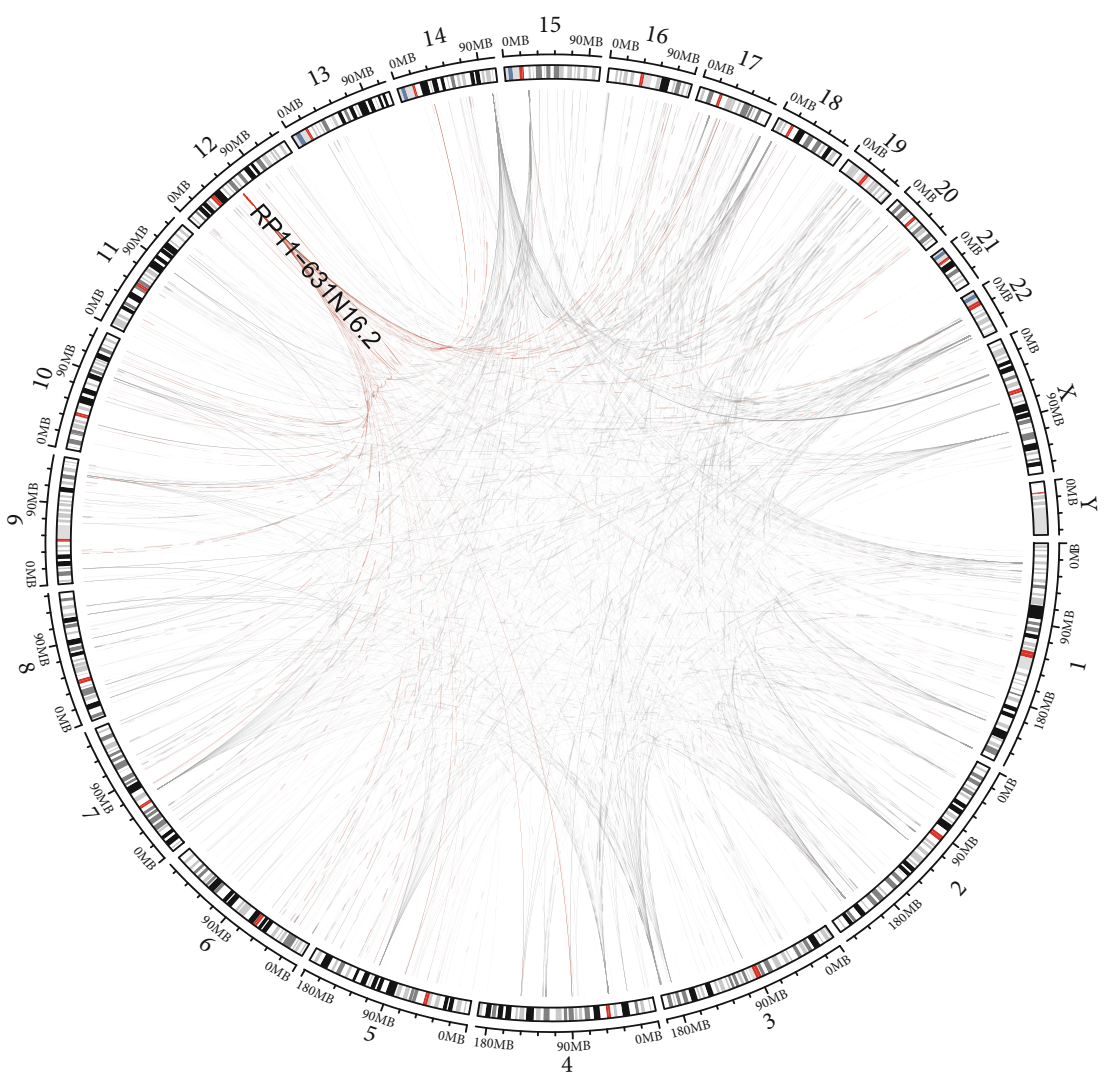

(b)

FIGURE 1: Differential ceRNA networks in ALS and control samples. 


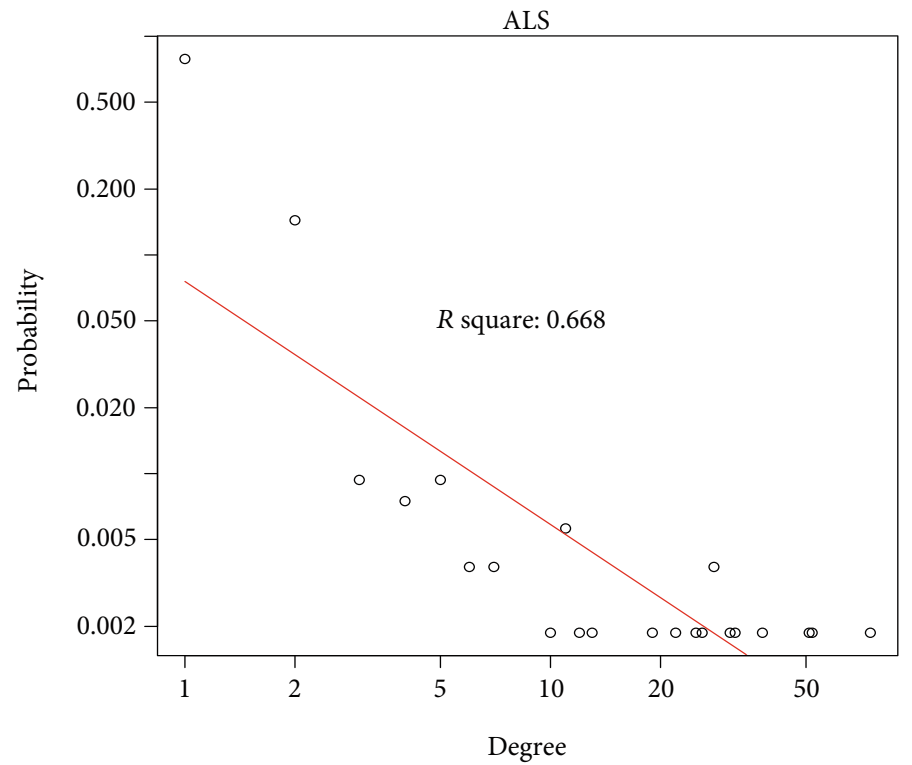

(a)

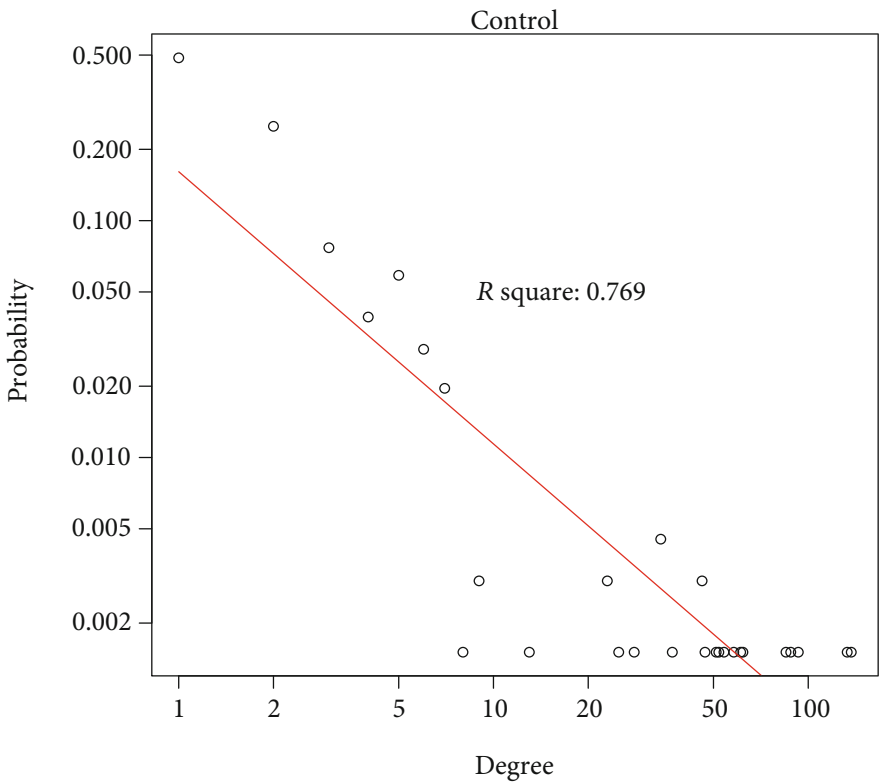

(b)

FIgURE 2: The degree distribution of the ceRNA network formed between lncRNA-mRNA in the ALS group and in the control group.

represents the ceRNA network in ALS patient samples, and Figure 1(b) represents the ceRNA network in control samples. We found that most lncRNAs and mRNAs were located on different chromosomes; in other words, the regulatory relationships among lncRNAs, mRNAs, and miRNAs involve trans mechanisms and miRNA sponging functions.

Further analysis was performed to characterize the degree distribution of the ceRNA network-formed lncRNAmRNA interactions. It was found that the degree distribution of the network approximately obeyed the power-law distribution: namely, the degree of most of the nodes was relatively small, and the degree of a small proportion of the nodes was relatively large (Figures 2(a) and 2(b)), which is in keeping with the nature of conventional bio- logical networks. There were some nodes with large degrees in the network. The nodes with relatively large degrees may play important roles in the network. For example, the lncRNAs MALAT1 and RP11-631N16.2 in the two networks of ALS and control samples may play important regulatory roles.

3.2. ceRNA Network Function Annotation. To study the functions associated with the ceRNA networks of ALS and control samples, the genes in these two networks were analysed with Metascape, and it was found that the genes in the ALS network were associated with GO biological processes such as autophagy (Figure 3(a)). Furthermore, some genes were the same between the regulatory networks of the ALS and 

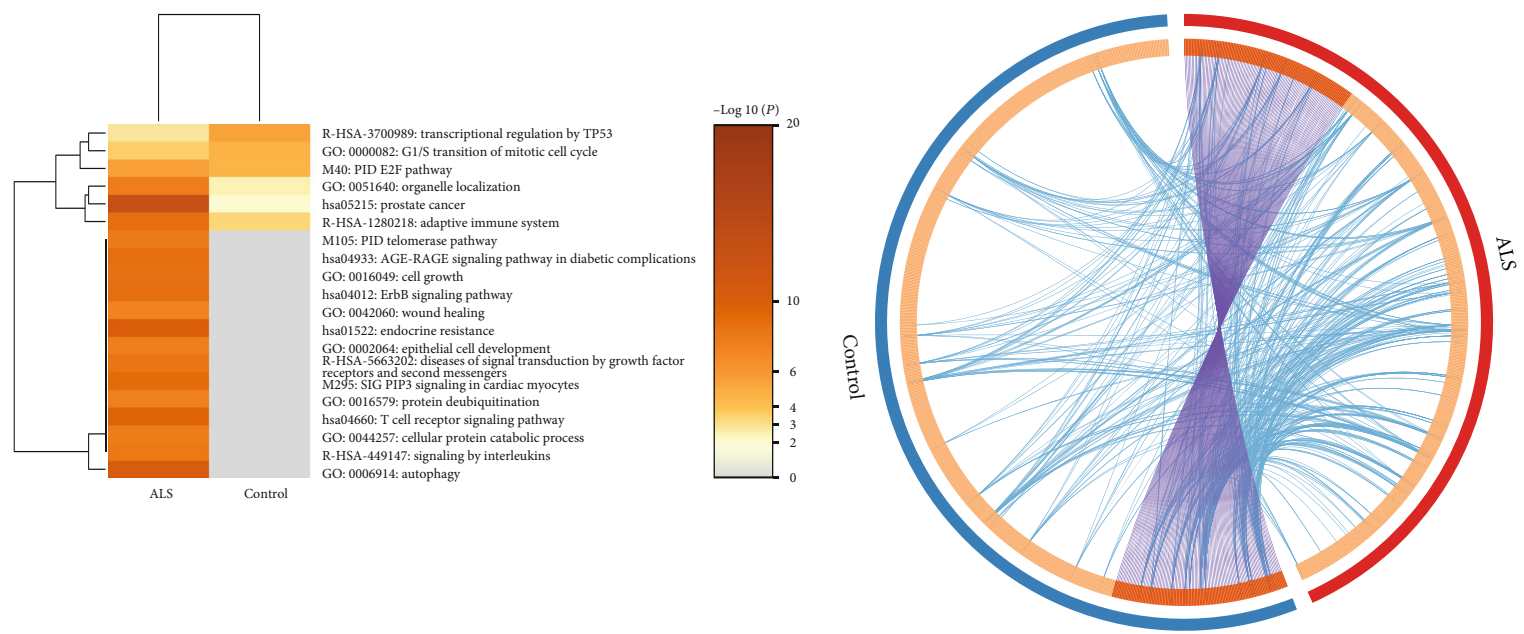

(a)

(b)
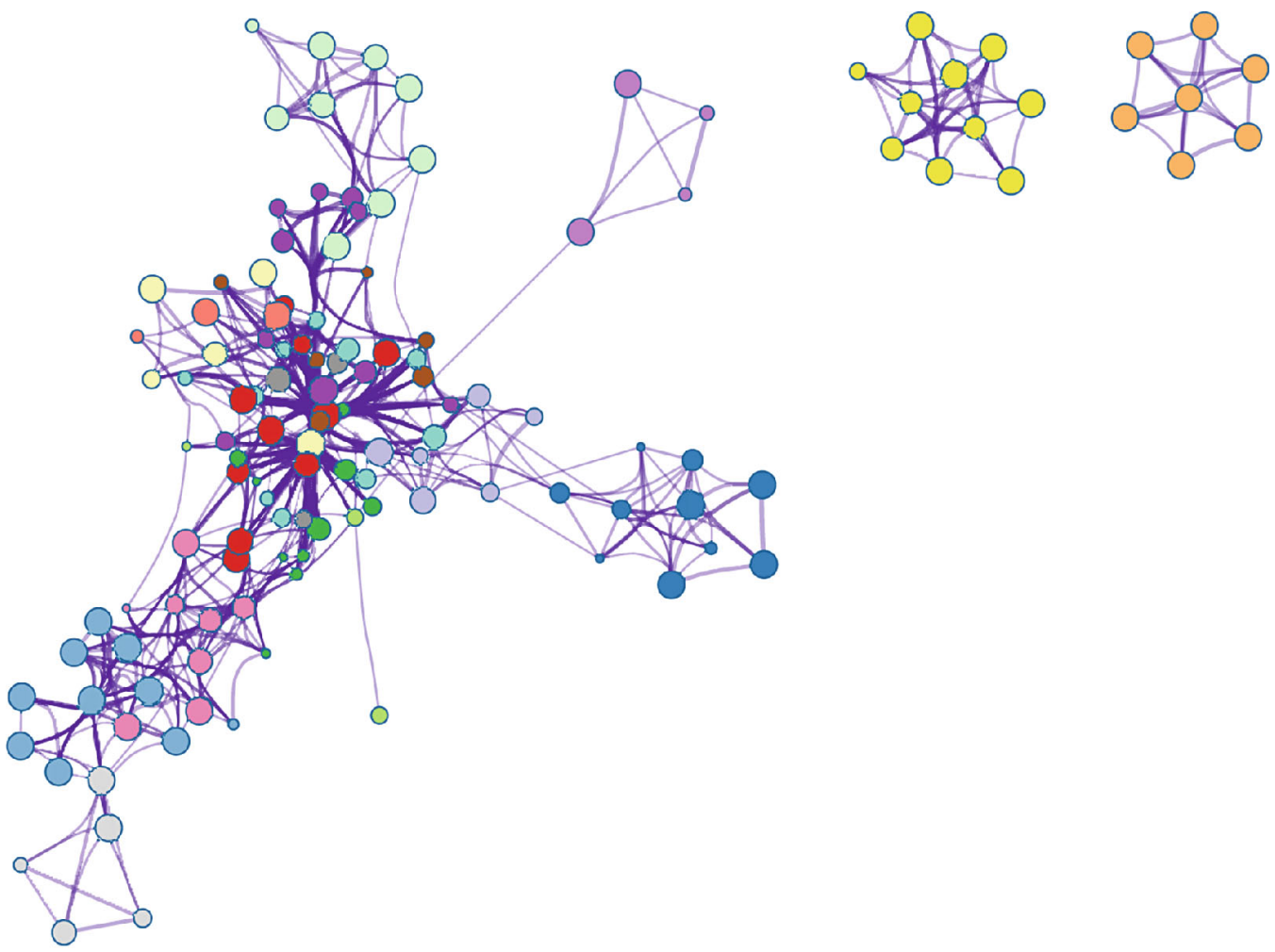
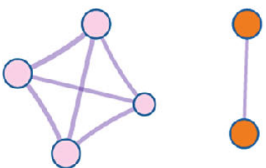

Prostate cancer

Autophagy

Endocrine resistance

$\mathrm{T}$ cell receptor signaling pathway

Adaptive immune system

Organelle localization

- SIG PIP3 signaling in cardiac myoctes

PID E2F pathway

AGE-RAGE signaling pathway in diabetic complications

ErbB signaling pathway
Cell growth

Diseases of signal transduction by growth factor receptors and second messenger

Signaling by interleukins

G1/S transition of mitotic cell cycle

Cellular protein catabolic process

PID telomerase pathway

Protein deubiquitination

Transcriptional regulation by TP53

Epithelial cell development

Wound healing

(c)

Figure 3: Continued. 


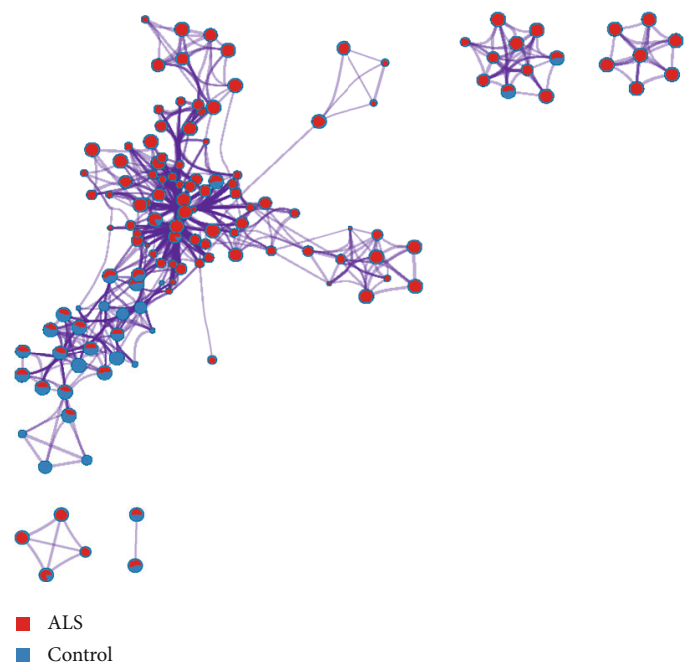

(d)

FIgURE 3: ceRNA network function annotation. (a) Biological processes associated with genes in the ceRNA network in ALS cases and control cases. (b) Comparison of 493 genes between ALS cases and control cases in the ceRNA network. The red/blue/light orange/dark orange colour represents ALS group genes/controls genes/differentially expressed genes/common genes, respectively. (c) Further analysis of the relationship among top 20 GO terms. (d) Most terms were enriched in different categories in the ALS group.

control samples, but most of the genes were different (Figure 3(b)). Some of the different genes were associated with common GO terms (Figure 3(b), blue line). Further analysis was performed to determine the relationships in the top 20 terms. Figure 3(c) shows the connections among the terms, where different colours indicate different categories. Compared with the terms for the control samples, most terms for the ALS samples were significantly enriched in different categories (Figure 3(d)).

3.3. Hub IncRNA Function in the Network. In both ALS and control samples, there were important hub nodes that played important roles in the networks. To study the functions of lncRNAs in ALS and control samples, the lncRNAs were functionally annotated with the genes they regulated. The lncRNAs were first analysed in the two ceRNA networks. MALAT1 was determined to serve as a miRNA sponge to regulate 75 genes in ALS samples (Figure 4(a)). Through functional enrichment analysis of these regulated genes, we found enrichment for the following terms: GO:0006623: protein targeting to vacuole, GO: 0016050: vesicle organization, hsa04064: $N F-\kappa B$ signalling pathway, and GO: 0006352: DNA-templated transcription and initiation (Figure 4(b)). Notably, it has been reported that some genes regulated by MALAT1 are involved in the pathogenesis of ALS, including DECR1 [26], CPEB4 [27], VPS37A [28], SP1 [10, 29], EEA1 [30], RB1 [31], and GCLC [32] (Figure 4(a)).

In control samples, RP11-631N16.2 was observed to exhibit an important function by regulating 137 genes (Figure 4(c)), and it was associated with GO terms, such as GO:0046467: membrane lipid biosynthetic process, and GO:0031647: regulation of protein stability (Figure 4(d)). STX4 (Figure 4(c)), one of the genes regulated by the lncRNA $R P 11-631 N 16.2$, encodes a protein that plays a vital role in the control regulation of glucose metabolism uptake in skel- etal muscle. Reductions in the STX4 protein expression levels lead to decreases in systemic hormone-stimulated glucose metabolism. Another gene regulated by the lncRNA RP11631N16.2 is CASP3 (Figure 4(c)), which encodes cysteineaspartic acid protease; this protein plays a central role in the execution phase of apoptosis, which is associated with neuronal death in Alzheimer's disease (AD).

3.4. PPIs Regulated by MALAT1. Since MALAT1 plays an important role in the network, it was hypothesized that the genes regulated by MALAT1 interact at the protein level. We depicted the PPI network of the genes regulated by MALAT1 using the STRING database (Figure 5(a)). The MCODE plug-in of Cytoscape was further used to mine the PPI modules, and it was determined that the modules formed by SYNRG, ITSN2, AAK1, PICALM, and AP3B1 may play important roles in ALS (Figure 5(b)).

\section{Discussion}

The typical manifestations of ALS are muscle weakness and atrophy, which severely affect the physical and mental health of patients. In this study, a number of meaningful findings were obtained regarding the pathogenesis of ALS. The lncRNAs in the ceRNA network have diverse functions and can regulate a variety of genes. $\mathrm{GO}$ analysis of genes regulated by MALAT1 revealed enrichment for several GO terms related to ALS.

In Figure 4(b), the terms GO:0006623: protein targeting to vacuole, and GO:0016050: vesicle organization, are depicted. A previous study has shown that miRNA signals in the plasma of ALS patients (PALS) can cross the bloodbrain barrier and enter the circulatory system [1]. Analysis of the differentially expressed miRNAs in extracellular vesicles (EVs) revealed elevated levels of 5 miRNAs and 


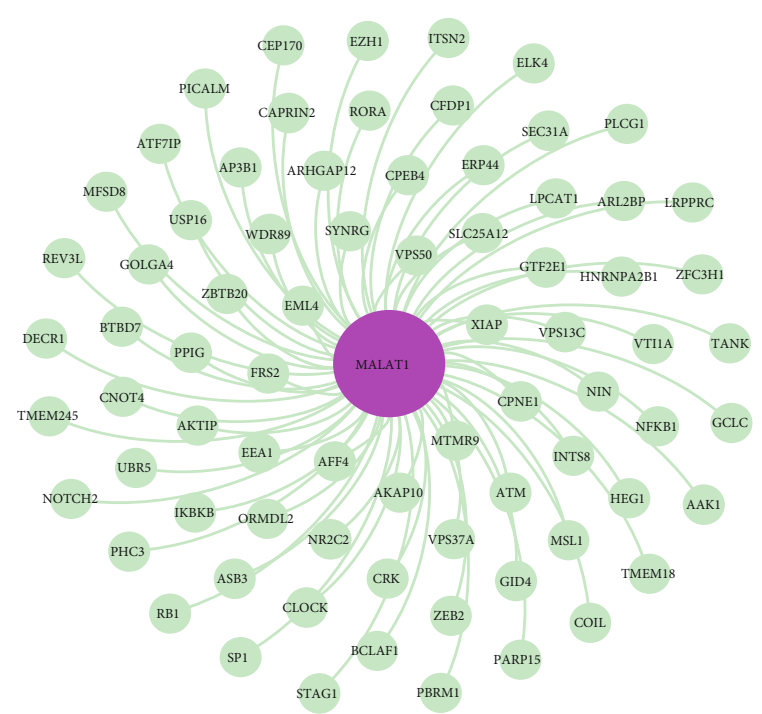

(a)

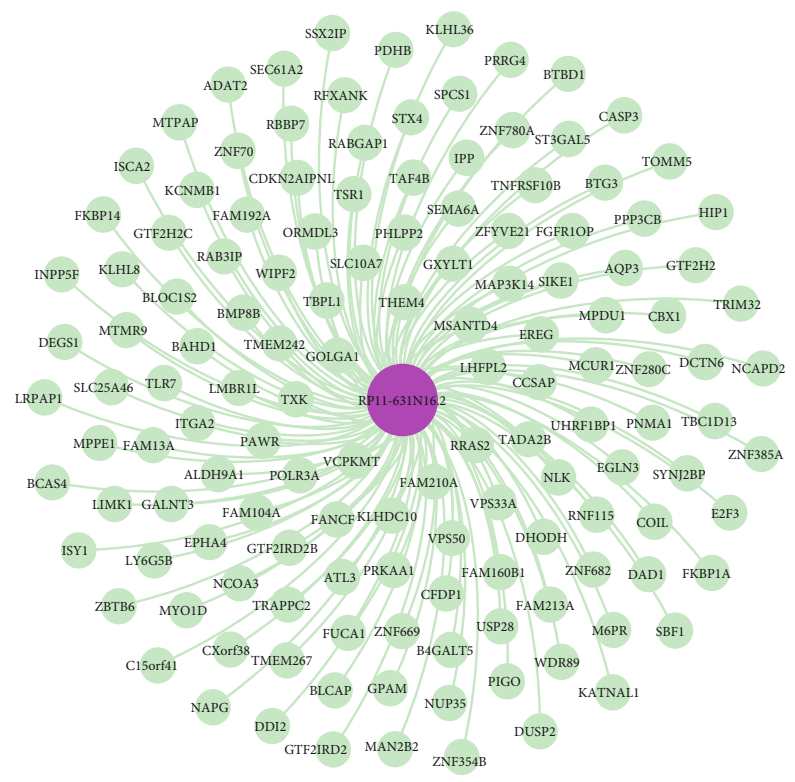

(c)

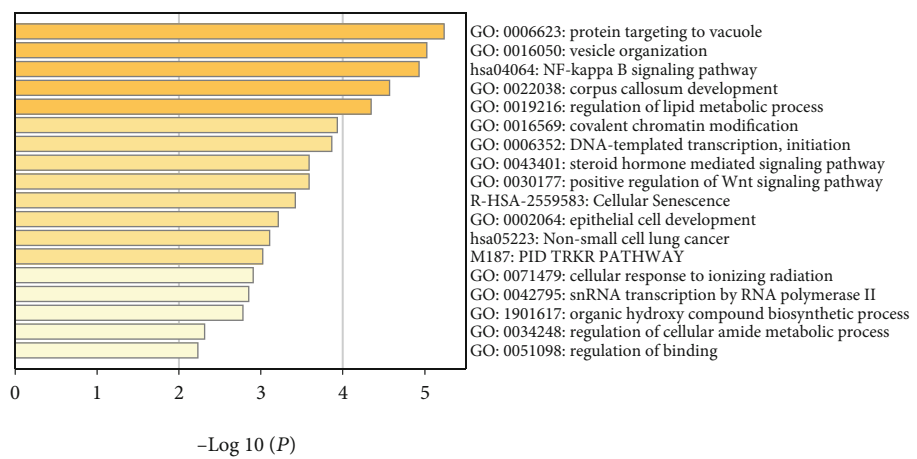

(b)

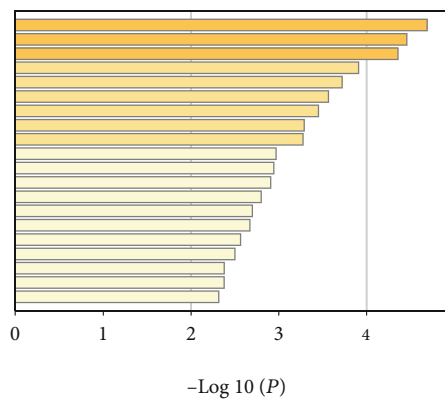

(d)

FIGURE 4: Hub lncRNA functions in the network: (a) seventy-five genes regulated by MALAT1 in ALS; (b) enriched GO terms for ALS patients in different categories; (c) one hundred thirty-seven genes regulated by RP11-631N16.2 in control samples; (d) enriched GO terms for control samples in different categories.

decreased levels of 22 miRNAs in EVs collected from PALS samples compared with control samples [1]. Four unregulated miRNAs associated with ALS involving miR-9-5p, miR-183-5p, miR-338-3p, and miR-1246 [1]. These results emphasize the diagnostic relevance of miR-15a-5p for distinguishing samples from healthy individuals from PALS samples and of miR-193a-5p for distinguishing among patients with low vs. high Revised ALS Functional Rating Scale (ALSFRS-R) scores [1]. The data in Table S2 suggest that miR-9-5p is related to the $A P 3 B 1$ and NF- $\kappa B$ genes, that miR-15a-5p is related to the SYNRG and AP3B1 genes, that miR-193a-5p is related to the TMEM245 gene, and that SYNRG, AP3B1, NF- $\kappa B$, and TMEM245 are regulated by MALAT1 (Figure 4(a)).
Another term depicted in Figure 4(b) is hsa04064: NF- $\kappa B$ signalling pathway. $N F-\kappa B$ is a pleiotropic transcription factor that exists in almost all cell types and is the terminus of a series of signal transduction events. Growth, tumorigenesis, and apoptosis are triggered by numerous stimuli related to many biological processes, such as inflammation and immunity. OPTN is known as an NF- $\kappa B$ basic regulationrelated protein and is involved in maintaining the morphology of the Golgi apparatus and in regulating exocytosis, endoplasmic reticulum stress, membrane receptor levels, type-I interferon response, cell death, and autophagy; nonsense and missense mutations of the OPTN gene abolish the inhibitory effect of NF- $\kappa B$ activation, and NF- $\kappa B$ inhibitors can be used to treat ALS $[6,13]$. 


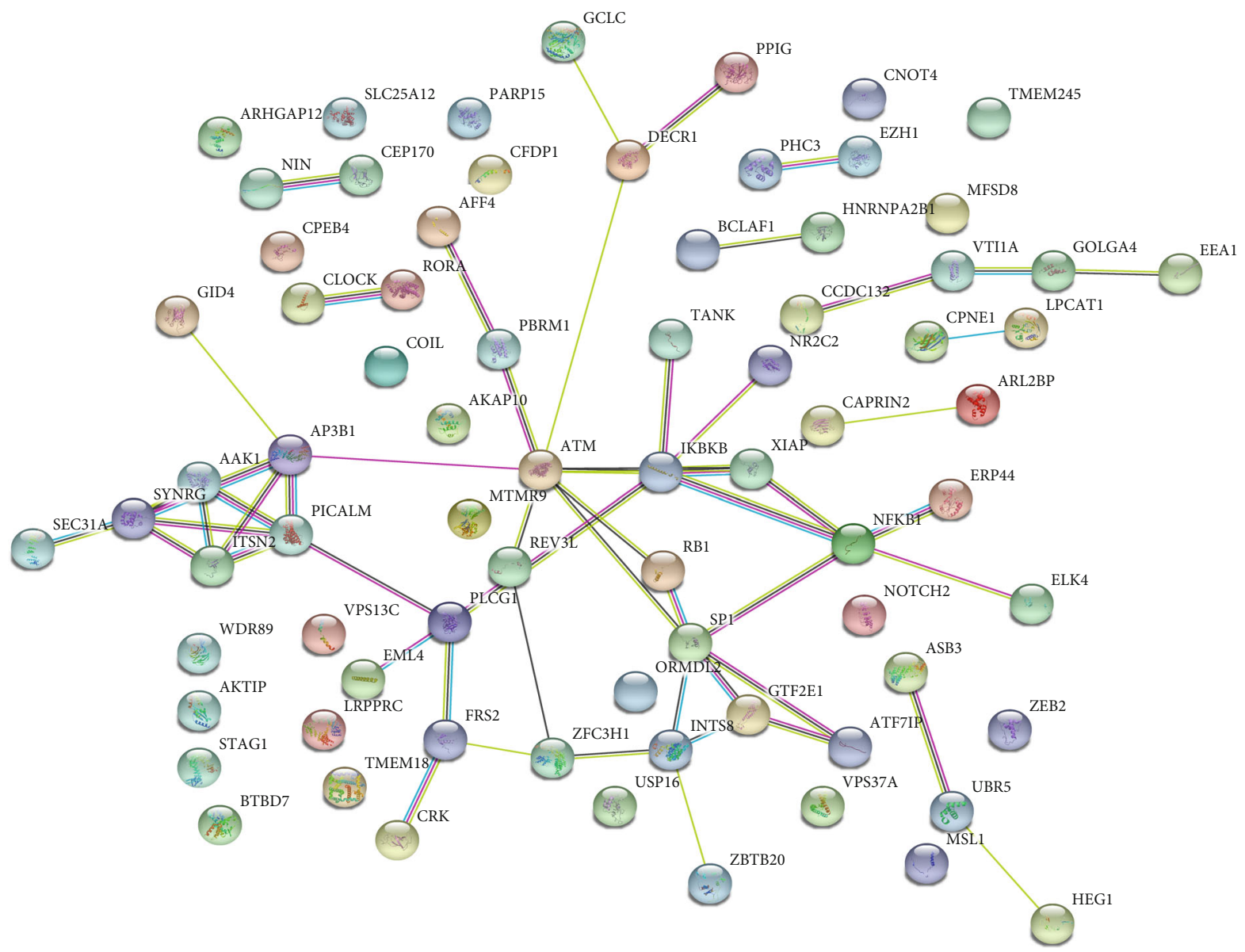

(a)

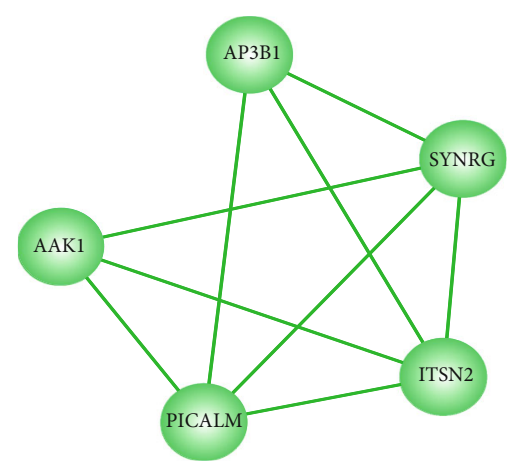

(b)

FIGURE 5: PPI network: (a) the red and green nodes represent the upregulated and downregulated genes, respectively; (b) modules formed by SYNRG, ITSN2, AAK1, PICALM, and AP3B1 in the PPI network.

A third term depicted in Figure 4(b) is GO:0006352: DNA-templated transcription and initiation. TDP-43 can bind to MALAT1. TDP-43 is a DNA/RNA binding protein encoded by the TARDBP gene that has been determined to be an ALS ubiquitination aggregate. Importantly, the given neuroinflammation is a pathological feature of ALS, and mutations to genes such as TARDBP enhance this neuroinflammation [7,33-36]. In addition, the MALAT-1-regulated gene $h n R N P A 2 / B 1$ encodes an RNA-binding protein that is associated with neurodegeneration $[16,37-40]$. Martinez et al. found that $h n R N P A 2 / B 1 D 290 \mathrm{~V}$ mutant fibroblasts and motor neurons differentiated from induced pluripotent stem cells obtained from ALS patients showed abnormal splicing changes and that the survival rates of induced pluripotent stem cell with mutations were decreased in long-term culture, which aggravated changes in gene expression and splicing, when placing stress on the cells [38].

Another gene regulated by MALAT1 is XIAP (shown in Figure 4(a)). Many studies [41-44] have shown that different pathways (including the mitochondrial apoptosis pathway, the glutamate excitotoxicity pathway, and the HGF overexpression pathway) increase the levels of apoptotic proteases 


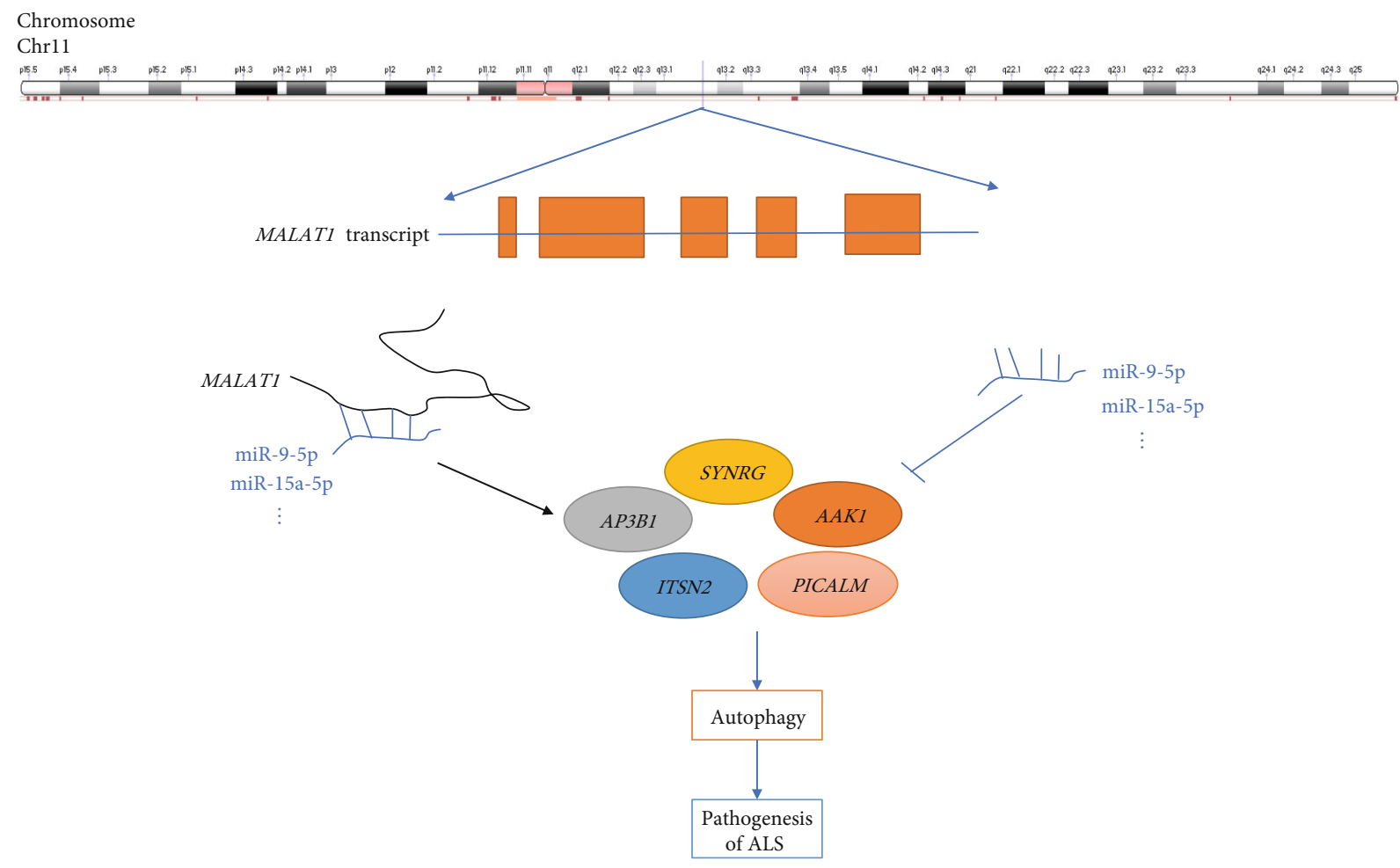

FIGURE 6: Potential mechanism of differentially expressed lncRNA sponges.

and decrease the expression levels of XIAP protein in the spinal cord, which is closely related to motor neuron degeneration in the process of ALS-related apoptosis due to mutant SOD-1. Importantly, changes in the expression levels of the XIAP protein may play important roles in the late stage of ALS.

The ATM gene is also regulated by MALAT1, as depicted in Figure 4(a). Studies $[45,46]$ have revealed that ATM plays an important role in the response to DNA damage observed in the pathogenesis of ALS. Moreover, neuronal dysfunction and neuronal death in ALS patients may be related to continuous DNA damage and the activation of the ATM and p53 proapoptotic signalling pathways.

Interestingly, in this study, the modules formed by SYNRG, ITSN2, PICALM, AP3B1, and AAK1 in the PPI network regulated by $M A L A T 1$ were closely related to one another (Figure 5(b)).

ITSN2, shown in Figure 5(b), is an adapter protein and a member of the conserved family of clathrin-mediated endocytosis proteins. ITSN2 may be involved in regulating the formation of clathrin-coated vesicles and may also participate in the maturation of clathrin-coated vesicles [47, 48].

$A A K 1$ is an adapter-related regulatory protein in the endocytic pathway of clathrin-coated vesicles. This protein selectively interacts with SOD1 mutants, rather than wildtype SOD1. Adapter receptor-associated protein complex 2 plays a role in receptor-mediated endocytosis, triggering clathrin assembly, interacting with membrane-bound receptors, and recruiting coding cofactors. The kinase activity of this complex is stimulated by clathrin or transcriptional splice variants, but its biological effectiveness has not been determined. The $A P 2 M 1 / M u 2$ subunit of adaptor protein complex
2 is phosphorylated to regulate clathrin-mediated endocytosis, which ensures the high-affinity binding of AP2 to cargo membrane proteins in the initial stage of endocytosis. Research on this gene in animal models has confirmed that abnormal functioning of components in the endoplasmic and synaptic vesicle recycling pathway is related to the pathology of ALS [49].

It has been reported in the literature that SYNRG gene mutations appear in the $17 q 12$ microdeletion syndrome, which is related to cognitive impairment and abnormal brain structure [50, 51]. AD and ALS are both neurodegenerative diseases, and PICALM has been proven to be the causative gene of $\mathrm{AD}$ [52-54]. In addition, there are a large number of published studies confirming that $A P 3 B 1$ is related to type 2 Hermansky-Pudlak syndrome [55-64]. Although these four genes (SYNRG, ITSN2, PICALM, and AP3B1) have not been reported in the literature on ALS, the SYNRG, ITSN2, PICALM, AP3B1, and AAK1 genes are regulated by $M A L A T 1$, and the proteins encoded by these genes are all related to clathrin. As far as we know, the clathrin may participate in the formation of early autophagosomes, and autophagy played a role in the pathogenesis of neurodegenerative diseases. Thus, we speculated that the PPI module formed by these five genes (SYNRG, ITSN2, PICALM, $A P 3 B 1$, and $A A K 1)$ is related to the autophagy and may play an important role in the pathogenesis of ALS.

\section{Conclusion}

In this study, we constructed a ceRNA network and found that isogenic regulation by MALAT1 may play an important role in the pathogenesis of ALS (Figure 6). We further 
observed that the modules formed by the MALAT1-regulated SYNRG, ITSN2, AAK1, PICALM, and AP3B1 ALS genes are potentially important roles of participants in the pathogenesis of ALS. Our findings provide a new perspective for understanding the mechanism underlying ALS. The AAK1encoding gene has been proven to be related to ALS, and further research is warranted to determine whether the other four genes are related to ALS.

\section{Data Availability}

Sample RNA-seq data (GSE115259) were downloaded from the Gene Expression Omnibus (GEO, http://www.ncbi.nlm .nih.gov/geo/) database.

\section{Conflicts of Interest}

The authors declare that the research was conducted in the absence of any commercial or financial relationships that could be construed as a potential conflict of interest.

\section{Authors' Contributions}

Dingsheng Liu, Xiaojia Zuo, and Peng Zhang contributed equally to this work.

\section{Acknowledgments}

The authors would like to express our heartfelt thanks to Dr. S.H. Zhou from the Nanjing University of Aeronautics and Astronautics for providing valuable help with this article. This work was supported by the National Natural Science Foundation of China (81772829 and 81830052), Key Projects for Collaborative Innovation of the Shanghai University of Medicine and Health Sciences, Construction Project of the Shanghai Key Laboratory of Molecular Imaging (18DZ2260400), Shanghai Municipal Education Commission (Class II Plateau Disciplinary Construction Program for Medical Technology of SUMHS, 2018-2020), Discipline Construction Program of the Pudong New District Health Bureau of Shanghai (Grant No. PWZzk2017-31), “Top-100 Talent Cultivation Plan" of Shanghai University of Medicine and Health Sciences, and Funding Scheme for Training Young Teachers in Shanghai Colleges (ZZJKYX19009).

\section{Supplementary Materials}

Supplementary 1. Figure S1: samples are separated by dimensionality reduction using the $t$-Distributed Stochastic Neighbour Embedding ( $t$-SNE) algorithm.

Supplementary 2. Table S1: potential lncRNA-mRNA ceRNA relationship pairs (Excel file).

Supplementary 3. Table S2: significant lncRNA-mRNA ceRNA relationship pairs.

\section{References}

[1] D. Saucier, G. Wajnberg, J. Roy et al., "Identification of a circulating miRNA signature in extracellular vesicles collected from amyotrophic lateral sclerosis patients," Brain Research, vol. 1708, pp. 100-108, 2019.

[2] E. P. McCann, L. Henden, J. A. Fifita et al., "Evidence for polygenic and oligogenic basis of Australian sporadic amyotrophic lateral sclerosis," Journal of Medical Genetics, vol. 58, pp. 8795, 2021.

[3] Y. Kosuge, H. Nango, H. Kasai et al., "Generation of cellular reactive oxygen species by activation of the EP2 receptor contributes to prostaglandin E2-induced cytotoxicity in motor neuron-like NSC-34 cells," Oxidative Medicine and Cellular Longevity, vol. 2020, Article ID 6101838, 14 pages, 2020.

[4] Y. J. Zhang, L. Guo, P. K. Gonzales et al., "Heterochromatin anomalies and double-stranded RNA accumulation underlie C9orf72 poly(PR) toxicity," Science, vol. 363, no. 6428, article eaav2606, 2019.

[5] A. Jimenez-Pacheco, J. M. Franco, S. Lopez et al., "Epigenetic mechanisms of gene regulation in amyotrophic lateral sclerosis," Advances in Experimental Medicine and Biology, vol. 978, pp. 255-275, 2017.

[6] H. Maruyama, H. Morino, H. Ito et al., "Mutations of optineurin in amyotrophic lateral sclerosis," Nature, vol. 465, no. 7295, pp. 223-226, 2010.

[7] D. R. Beers and S. H. Appel, "Immune dysregulation in amyotrophic lateral sclerosis: mechanisms and emerging therapies," The Lancet Neurology, vol. 18, no. 2, pp. 211-220, 2019.

[8] A. Burberry, M. F. Wells, F. Limone et al., "C9orf72 suppresses systemic and neural inflammation induced by gut bacteria," Nature, vol. 582, no. 7810, pp. 89-94, 2020.

[9] C. S. Petit, J. J. Lee, S. Boland et al., "Inhibition of sphingolipid synthesis improves outcomes and survival in GARP mutant wobbler mice, a model of motor neuron degeneration," Proceedings of the National Academy of Sciences of the United States of America, vol. 117, no. 19, pp. 10565-10574, 2020.

[10] M. P. Cuajungco and G. J. Lees, "Zinc metabolism in the brain: relevance to human neurodegenerative disorders," Neurobiology of Disease, vol. 4, no. 3-4, pp. 137-169, 1997.

[11] H. X. Deng, W. Chen, S. T. Hong et al., "Mutations in UBQLN2 cause dominant X-linked juvenile and adult-onset ALS and ALS/dementia," Nature, vol. 477, no. 7363, pp. 211215, 2011.

[12] P. A. Dion, H. Daoud, and G. A. Rouleau, "Genetics of motor neuron disorders: new insights into pathogenic mechanisms," Nature Reviews Genetics, vol. 10, no. 11, pp. 769-782, 2009.

[13] H. Maruyama and H. Kawakami, "Optineurin and amyotrophic lateral sclerosis," Geriatrics \& Gerontology International, vol. 13, no. 3, pp. 528-532, 2013.

[14] C. H. Wu, C. Fallini, N. Ticozzi et al., "Mutations in the profilin 1 gene cause familial amyotrophic lateral sclerosis," Nature, vol. 488, no. 7412, pp. 499-503, 2012.

[15] A. Osmanovic, M. Widjaja, A. Förster et al., "SPG7 mutations in amyotrophic lateral sclerosis: a genetic link to hereditary spastic paraplegia," Journal of Neurology, vol. 267, no. 9, pp. 2732-2743, 2020.

[16] Y. C. Xue, C. S. Ng, P. Xiang et al., "Dysregulation of RNAbinding proteins in amyotrophic lateral sclerosis," Frontiers in Molecular Neuroscience, vol. 13, p. 78, 2020.

[17] R. Waller, M. Wyles, P. R. Heath et al., "Small RNA sequencing of sporadic amyotrophic lateral sclerosis cerebrospinal fluid reveals differentially expressed miRNAs related to neural and glial activity," Frontiers in Neuroscience, vol. 11, p. 731, 2017. 
[18] Y. P. Chen, Q. Q. Wei, X. P. Chen et al., "Aberration of miRNAs expression in leukocytes from sporadic amyotrophic lateral sclerosis," Frontiers in Molecular Neuroscience, vol. 9, p. 69, 2016.

[19] E. Dardiotis, A. M. Aloizou, V. Siokas et al., "The role of microRNAs in patients with amyotrophic lateral sclerosis," Journal of Molecular Neuroscience, vol. 66, no. 4, pp. 617628, 2018.

[20] A. M. Helferich, S. J. Brockmann, J. Reinders et al., "Dysregulation of a novel miR-1825/TBCB/TUBA4A pathway in sporadic and familial ALS," Cellular and Molecular Life Sciences, vol. 75, no. 23, pp. 4301-4319, 2018.

[21] S. Gagliardi, S. Zucca, C. Pandini et al., "Long non-coding and coding RNAs characterization in peripheral blood mononuclear cells and spinal cord from amyotrophic lateral sclerosis patients," Scientific Reports, vol. 8, no. 1, article 2378, 2018.

[22] M. Katsu, Y. Hama, J. Utsumi et al., "MicroRNA expression profiles of neuron-derived extracellular vesicles in plasma from patients with amyotrophic lateral sclerosis," Neuroscience Letters, vol. 708, article 134176, 2019.

[23] B. de Felice, M. Guida, M. Guida, C. Coppola, G. de Mieri, and R. Cotrufo, "A miRNA signature in leukocytes from sporadic amyotrophic lateral sclerosis," Gene, vol. 508, no. 1, pp. 3540, 2012 .

[24] L. Salmena, L. Poliseno, Y. Tay, L. Kats, and P. P. Pandolfi, “A ceRNA hypothesis: the Rosetta stone of a hidden RNA language?," Cell, vol. 146, no. 3, pp. 353-358, 2011.

[25] D. S. Sardina, S. Alaimo, A. Ferro, A. Pulvirenti, and R. Giugno, "A novel computational method for inferring competing endogenous interactions," Briefings in Bioinformatics, vol. 18, no. 6, pp. 1071-1081, 2017.

[26] C. Tunca, T. Şeker, F. Akçimen et al., "Revisiting the complex architecture of ALS in Turkey: expanding genotypes, shared phenotypes, molecular networks, and a public variant database," Human Mutation, vol. 41, no. 8, pp. e7-e45, 2020.

[27] J. Shin, J. S. Salameh, and J. D. Richter, "Impaired neurodevelopment by the low complexity domain of CPEB4 reveals a convergent pathway with neurodegeneration," Scientific Reports, vol. 6, no. 1, article 29395, 2016.

[28] C. J. Shoemaker, T. Q. Huang, N. R. Weir, N. J. Polyakov, S. W. Schultz, and V. Denic, "CRISPR screening using an expanded toolkit of autophagy reporters identifies TMEM41B as a novel autophagy factor," PLoS biology, vol. 17, no. 4, article e2007044, 2019.

[29] V. García-Morales, G. Rodríguez-Bey, L. Gómez-Pérez et al., "Sp1-regulated expression of $p 11$ contributes to motor neuron degeneration by membrane insertion of TASK1," Nature Communications, vol. 10, no. 1, article 3784, 2019.

[30] A. Otomo, S. Hadano, T. Okada et al., "ALS2, a novel guanine nucleotide exchange factor for the small GTPase Rab5, is implicated in endosomal dynamics," Human Molecular Genetics, vol. 12, no. 14, pp. 1671-1687, 2003.

[31] J. L. Gonzalez de Aguilar, C. Niederhauser-Wiederkehr, B. Halter et al., "Gene profiling of skeletal muscle in an amyotrophic lateral sclerosis mouse model," Physiological Genomics, vol. 32, no. 2, pp. 207-218, 2008.

[32] C. Zhang, Y. Yang, W. Liang et al., "Neuroprotection by urate on the mutant hSOD1-related cellular and Drosophila models of amyotrophic lateral sclerosis: implication for GSH synthesis via activating $A k t / G S K 3 \beta / N r f 2 / G C L C$ pathways," Brain Research Bulletin, vol. 146, pp. 287-301, 2019.
[33] J. R. Tollervey, T. Curk, B. Rogelj et al., "Characterizing the RNA targets and position-dependent splicing regulation by TDP-43," Nature Neuroscience, vol. 14, no. 4, pp. 452-458, 2011.

[34] C. Lagier-Tourenne and D. W. Cleveland, "Rethinking ALS: the FUS about TDP-43," Cell, vol. 136, no. 6, pp. 1001-1004, 2009.

[35] A. C. Elden, H. J. Kim, M. P. Hart et al., "Ataxin-2 intermediate-length polyglutamine expansions are associated with increased risk for ALS," Nature, vol. 466, no. 7310, pp. 1069-1075, 2010.

[36] E. Kabashi, P. N. Valdmanis, P. Dion et al., “TARDBP mutations in individuals with sporadic and familial amyotrophic lateral sclerosis," Nature Genetics, vol. 40, no. 5, pp. 572-574, 2008.

[37] H. J. Kim, N. C. Kim, Y. D. Wang et al., "Mutations in prionlike domains in $h n R N P A 2 B 1$ and $h n R N P A 1$ cause multisystem proteinopathy and ALS," Nature, vol. 495, no. 7442, pp. 467473, 2013.

[38] F. J. Martinez, G. A. Pratt, E. L. van Nostrand et al., "ProteinRNA networks regulated by Normal and ALS-associated mutant HNRNPA2B1 in the nervous system," Neuron, vol. 92, no. 4, pp. 780-795, 2016.

[39] X. Qi, Q. Pang, J. Wang et al., "Familial early-onset Paget's disease of bone associated with a novel hnRNPA2B1 mutation," Calcified Tissue International, vol. 101, no. 2, pp. 159-169, 2017.

[40] M. Seelen, A. E. Visser, D. J. Overste et al., "No mutations in $h n R N P A 1$ and $h n R N P A 2 B 1$ in Dutch patients with amyotrophic lateral sclerosis, frontotemporal dementia, and inclusion body myopathy," Neurobiology of Aging, vol. 35, no. 8, pp. 1956.e9-1956.e11, 2014.

[41] H. Inoue, K. Tsukita, T. Iwasato et al., "The crucial role of caspase- 9 in the disease progression of a transgenic ALS mouse model," The EMBO Journal, vol. 22, no. 24, pp. 6665-6674, 2003.

[42] K. Kadoyama, H. Funakoshi, W. Ohya, and T. Nakamura, "Hepatocyte growth factor (HGF) attenuates gliosis and motoneuronal degeneration in the brainstem motor nuclei of a transgenic mouse model of ALS," Neuroscience Research, vol. 59, no. 4, pp. 446-456, 2007.

[43] J. S. Pyo, Y. S. Ko, W. H. Kim et al., "Impairment of nuclear factor-kappaB activation increased glutamate excitotoxicity in a motoneuron-neuroblastoma hybrid cell line expressing mutant (G93A) Cu/Zn-superoxide dismutase," Journal of Neuroscience Research, vol. 88, no. 11, pp. 2494-2503, 2010.

[44] E. Tokuda, S. Ono, K. Ishige et al., "Dysequilibrium between caspases and their inhibitors in a mouse model for amyotrophic lateral sclerosis," Brain Research, vol. 1148, pp. 234-242, 2007.

[45] Y. Q. Yang, Y. H. Zheng, C. T. Zhang et al., "Wild-type p53induced phosphatase 1 down-regulation promotes apoptosis by activating the DNA damage-response pathway in amyotrophic lateral sclerosis," Neurobiology of Disease, vol. 134, article 104648, 2020.

[46] B. W. Kim, Y. E. Jeong, M. Wong, and L. J. Martin, "DNA damage accumulates and responses are engaged in human ALS brain and spinal motor neurons and DNA repair is activatable in iPSC-derived motor neurons with SOD1 mutations," Acta Neuropathologica Communications, vol. 8, no. 1, p. 7, 2020. 
[47] A. E. Rodriguez-Fraticelli, S. Vergarajauregui, D. J. Eastburn et al., "The $C d c 42$ GEF intersectin 2 controls mitotic spindle orientation to form the lumen during epithelial morphogenesis," The Journal of Cell Biology, vol. 189, no. 4, pp. 725-738, 2010.

[48] A. S. Sengar, J. Ellegood, A. P. Yiu et al., "Vertebrate intersectin1 is repurposed to facilitate cortical midline connectivity and higher order cognition," The Journal of Neuroscience, vol. 33, no. 9, pp. 4055-4065, 2013.

[49] B. Shi, S. D. Conner, and J. Liu, "Dysfunction of endocytic kinase AAK1 in ALS," International Journal of Molecular Sciences, vol. 15, no. 12, pp. 22918-22932, 2014.

[50] N. W. Hendrix, M. Clemens, T. P. Canavan, U. Surti, and A. Rajkovic, "Prenatally diagnosed $17 q 12$ microdeletion syndrome with a novel association with congenital diaphragmatic hernia," Fetal Diagnosis and Therapy, vol. 31, no. 2, pp. 129133, 2012.

[51] H. Yamamoto, F. Hayakawa, T. Yasuda et al., "ZNF384-fusion proteins have high affinity for the transcriptional coactivator EP300 and aberrant transcriptional activities," FEBS Letters, vol. 593, no. 16, pp. 2151-2161, 2019.

[52] D. Harold, R. Abraham, P. Hollingworth et al., "Genome-wide association study identifies variants at CLU and PICALM associated with Alzheimer's disease," Nature Genetics, vol. 41, no. 10, pp. 1088-1093, 2009.

[53] S. Seshadri, A. L. Fitzpatrick, M. A. Ikram et al., "Genome-wide analysis of genetic loci associated with Alzheimer disease," JAMA, vol. 303, no. 18, pp. 1832-1840, 2010.

[54] M. A. van Es and L. H. van den Berg, "Alzheimer's disease beyond APOE," Nature Genetics, vol. 41, no. 10, pp. 10471048, 2009.

[55] R. Jing, Y. Kong, G. Han et al., "The mutation of the Ap3b1 gene causes uterine hypoplasia in pearl mice," Reproductive Sciences, vol. 27, no. 1, pp. 182-191, 2020.

[56] E. Gonçalves, A. Fragoulis, L. Garcia-Alonso, T. Cramer, J. Saez-Rodriguez, and P. Beltrao, "Widespread posttranscriptional attenuation of genomic copy-number variation in cancer," Cell systems, vol. 5, no. 4, pp. 386-398.e4, 2017.

[57] M. de Boer, K. van Leeuwen, J. Geissler et al., "HermanskyPudlak syndrome type 2: aberrant pre-mRNA splicing and mislocalization of granule proteins in neutrophils," Human Mutation, vol. 38, no. 10, pp. 1402-1411, 2017.

[58] R. Jing, H. Zhang, Y. Kong et al., "Different functions of biogenesis of lysosomal organelles complex 3 subunit 1 (Hps1) and adaptor-related protein complex 3 , beta 1 subunit (Ap3b1) genes on spermatogenesis and male fertility," Reproduction, Fertility, and Development, vol. 31, no. 5, pp. 972982, 2019.

[59] E. Karampini, M. Schillemans, M. Hofman et al., "Defective AP-3-dependent VAMP8 trafficking impairs Weibel-Palade body exocytosis in Hermansky-Pudlak syndrome type 2 blood outgrowth endothelial cells," Haematologica, vol. 104, no. 10, pp. 2091-2099, 2019.

[60] Y. Miao, H. Y. Zhu, C. Qiao et al., "Pathogenic gene mutations or variants identified by targeted gene sequencing in adults with hemophagocytic lymphohistiocytosis," Frontiers in Immunology, vol. 10, p. 395, 2019.

[61] E. Mukda, O. Trachoo, E. Pasomsub et al., "Exome sequencing for simultaneous mutation screening in children with hemophagocytic lymphohistiocytosis," International Journal of Hematology, vol. 106, no. 2, pp. 282-290, 2017.
[62] T. Nishikawa, K. Okamura, M. Moriyama et al., "Novel AP3B1 compound heterozygous mutations in a Japanese patient with Hermansky-Pudlak syndrome type 2," The Journal of Dermatology, vol. 47, no. 2, pp. 185-189, 2020.

[63] T. S. Rubin, K. Zhang, C. Gifford et al., "Perforin and CD107a testing is superior to NK cell function testing for screening patients for genetic HLH," Blood, vol. 129, no. 22, pp. 29932999, 2017.

[64] Y. Schaletzki, M. L. Kromrey, S. Bröderdorf et al., "Several adaptor proteins promote intracellular localisation of the transporter MRP4/ABCC4 in platelets and haematopoietic cells," Thrombosis and Haemostasis, vol. 117, no. 1, pp. 105$115,2017$. 